\title{
Severe Maxillofacial Infection After Implant Crown Recementation: A Case Report
}

\author{
Sung-Ouk Kim, DDS, MSD' ${ }^{1}$ Kwan-Soo Park, DDS, MSD, PhD \\ ${ }^{1}$ Resident, Department of Oral and Maxillofacial Surgery, Inje University Sanggye-Paik Hospital, College of \\ Medicine, Inje University, Seoul, Korea \\ ${ }^{2}$ Associate professor, Department of Oral and Maxillofacial Surgery, Inje University Sanggye-Paik Hospital, \\ College of Medicine, Inje University, Seoul, Korea \\ *Corresponding author: Kwan-Soo Park, Department of Oral and Maxillofacial Surgery, Inje University \\ Sanggye-Paik Hospital, 1342, Dongil-ro, Nowon-gu, Seoul 01757, Korea \\ Tel : +82-2-950-1161. Fax :+82-2-950-1167.E-mail : OMS_kspark@paik.ac.kr
}

\section{Abstract}

Maxillofacial infections from odontogenic or non-odontogenic causes can extend to the fascial space, a potential space formed by the fascia. Although morbidity due to infection has been considerably reduced because of modern antibiotic therapy, it can be life-threatening when the infection invades the deep fascial space. There are various causes of infection in the maxillofacial region, mostly following odontogenic or invasive dental treatments. We report a case of severe infection of the fascial spaces and deep neck after non-invasive dental treatment involving recementation of the implant crown in a male in his 60s, with reporting on its causes and treatment options.

Keywords: Deep neck, Emphysema, Fascia, Infection, Peri-Implantitis

pISSN 2765-7833

eISSN 2765-7841

Journal of implantology and applied sciences 2021; $25(3): 132-140$

https://doi.org/10.32542/implantology.2021014

Received: August 2, 2021

Revised: August 26, 2021

Accepted: September 6, 2021

\section{ORCID}

\section{Sung-Ouk Kim}

https://orcid.org/0000-0001-8052-4282

Kwan-Soo Park

https://orcid.org/0000-0002-0254-279X

Copyright $($ 2021. The Korean Academy of Oral \& Maxillofacial Implantology

\footnotetext{
This is an Open Access article distributed under the terms of the Creative Commons Attribution Non-Commercial License (http://creativecommons. org/licenses/by-nc/4.0/) which permits unrestricted non-commercial use, distribution, and reproduction in any medium, provided the original work is properly cited.
}

\section{I . Introduction}

The fascial space is a potential space surrounded by fascia; it does not exist under normal circumstances but is formed because of an infection. ${ }^{1}$ When an odontogenic infection spreads to the maxillofacial fascial space through the alveolar bone and soft tissue, it can rapidly spread further as there is no anatomical protection. The infection can also spread to adjacent areas, such as the deep neck, owing to muscle contraction and relaxation.

Since the fascial spaces are interconnected, multiple fascial spaces are often affected; when the infection invades the deeper fascial spaces, it can cause complications, such as acute airway obstruction, sepsis, mediastinitis, cavernous sinus thrombosis, and necrotizing fasciitis, and thus can be life-threatening.

Maxillofacial fascial space infection can have odontogenic and non-odontogenic causes. Odontogenic infections, such as dental caries and periodontitis, are the main causes. The most common form of odontogenic infection is apical infection, which occurs when 
bacteria invade the root canal of a tooth. Such fascial space infections may occur after invasive dental treatment, as in the case of secondary infection after tooth extraction; however, they may also occur after non-invasive dental treatment. Posttreatment fascial space infections have not been widely discussed. Moreover, in implant-related treatment, little is known about severe fascial space infection associated with a non-surgical, non-invasive procedure.

Here, we report the case of a patient with severe fascial space infection that occurred after implant prosthesis recementation, which is a non-invasive procedure.

\section{II . Case Report}

A male patient in his $60 \mathrm{~s}$ with a history of hypertension, diabetes, and hyperlipidemia was admitted to our emergency room. One week prior, the implant prosthesis in his right second molar in the mandible had fallen off and was recemented. After treatment, the patient was admitted to the emergency room of the hospital because of swelling of the right mandibular posterior gingiva and neck, which were the main complaints (Fig. 1). Bone resorption patterns were observed around the implant in the right second molar of the mandible in the panoramic view (Fig. 2). At the time of admission to the emergency room, his vital signs were as follows: blood pressure, $146 / 66 \mathrm{mmHg}$; body temperature, $36.4^{\circ} \mathrm{C}$; and pulse rate,

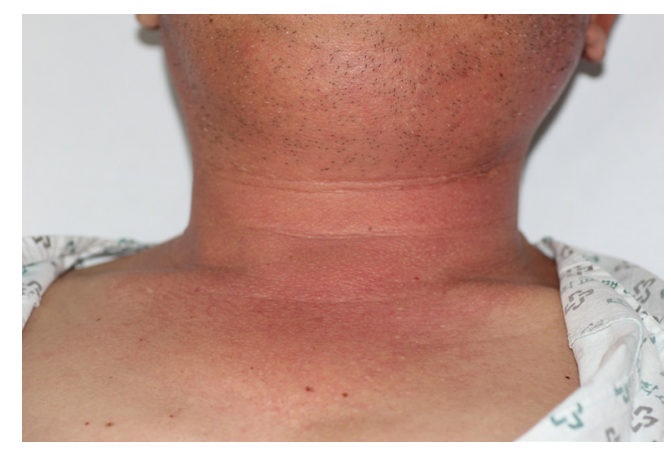

Fig. 1. First visit clinical photo: redness and swelling of submandibular area and neck were seen.

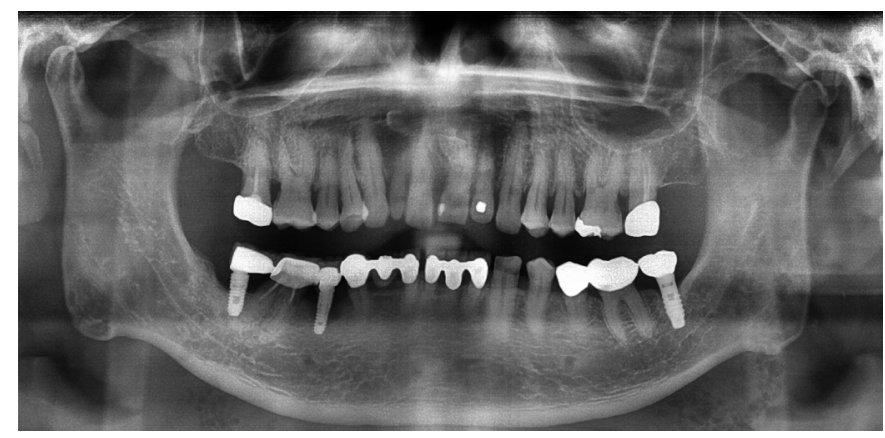

Fig. 2. First visit panoramic view: mild bone loss on mandibular right second molar implant area. 
97 beats/min. Laboratory test results were as follows: white blood cell (WBC) count, 16,750 $\mu \mathrm{L}$; creatinine level, $1.38 \mathrm{mg} / \mathrm{dL}$; and C-reactive protein (CRP) level, $37.8 \mathrm{mg} / \mathrm{L}$.

At the time of admission, non-enhanced computed tomography (CT) was performed because of high creatinine levels and a history of taking metformin-based diabetic drugs. Imaging revealed an air bubblefilled cavity extending from the right sublingual space through the submental space to the left sublingual space, as well as in the left submandibular space and left parapharyngeal space extending to the inner side of the mandibular condyle. In addition, swelling in the left sublingual space caused airway narrowing (Fig. 3). Magnetic resonance imaging (MRI) showed hyperintensity in the left submandibular and parapharyngeal spaces and bilateral sublingual spaces on T2-weighted images (Fig. 4). Bilateral
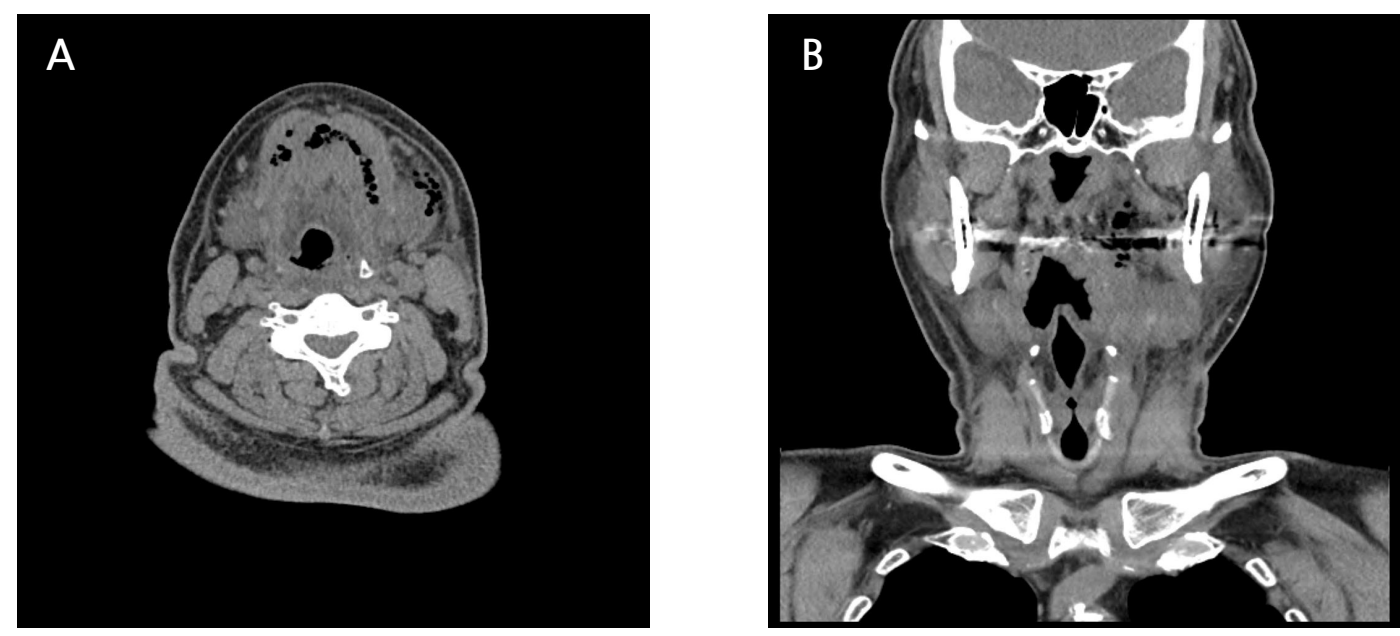

Fig. 3. Preoperative non-enhanced computed tomography (CT). (A) axial view: air bubble-filled cavity is seen on both sublingual and submental spaces, (B) coronal view: air bubble-filled cavity is seen on the left parapharyngeal space.
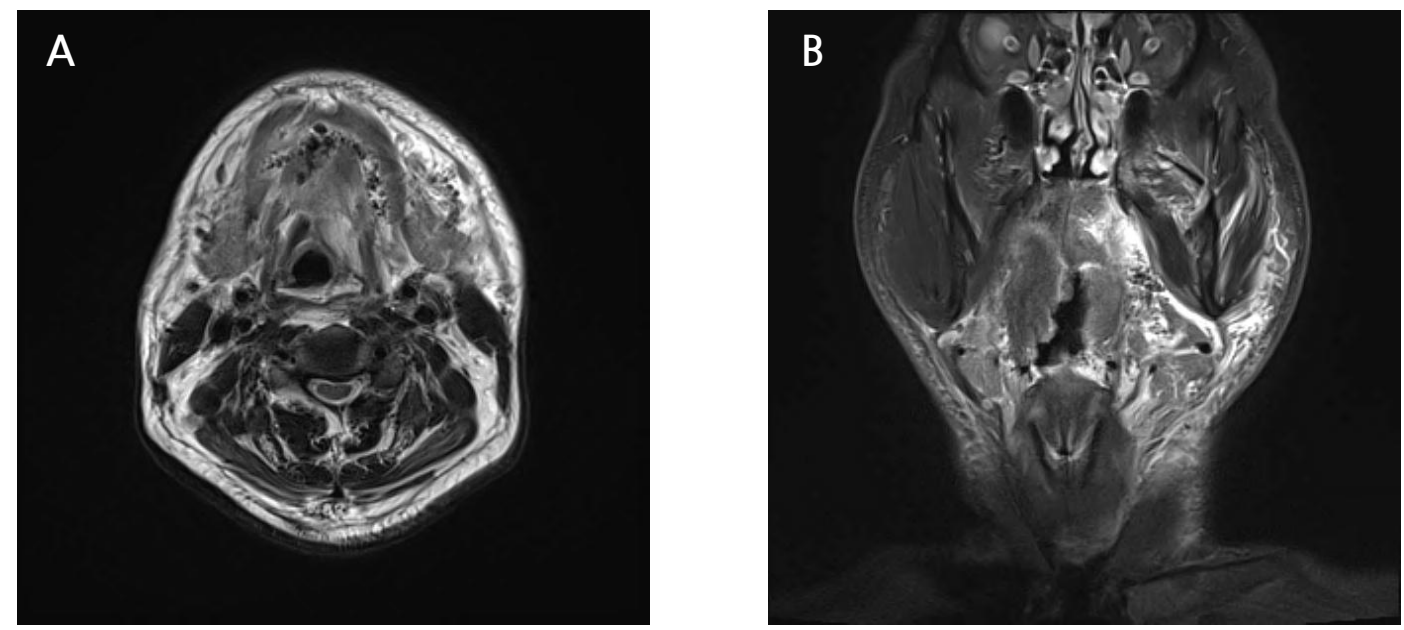

Fig. 4. Preoperative magnetic resonance imaging. (A) T2 axial view: Hypersignal intensity is seen on both sublingual and submental spaces, (B) T2 coronal view: Hypersignal intensity is seen on the left parapharyngeal space. 
sublingual space, left parapharyngeal space, left submandibular space, and submental space abscesses were diagnosed. The pus was drained by approaching the bilateral sublingual space and left submandibular space through a skin incision in the submental area under general anesthesia and approaching the left parapharyngeal space through an intraoral incision (Fig. 5).

The WBC and CRP levels 4 days after the first surgery decreased to $13,770 \mu \mathrm{L}$ and $6.6 \mathrm{mg} / \mathrm{dL}$, respectively, with reduction of symptoms such as swelling and tenderness. However, neck redness and pain persisted; hence, additional imaging tests were performed. The creatinine level improved to the normal range, and contrast-enhanced CT was performed. Imaging revealed pus cavities in the left masticator, submandibular, sublingual, parapharyngeal, and right visceral spaces (Fig. 6). A second surgery was performed under general anesthesia, and the left parapharyngeal space, left submandibular space, and right visceral space were drained, and a vacuum drain was maintained in the right visceral space for continuous pus drainage (Fig. 7).

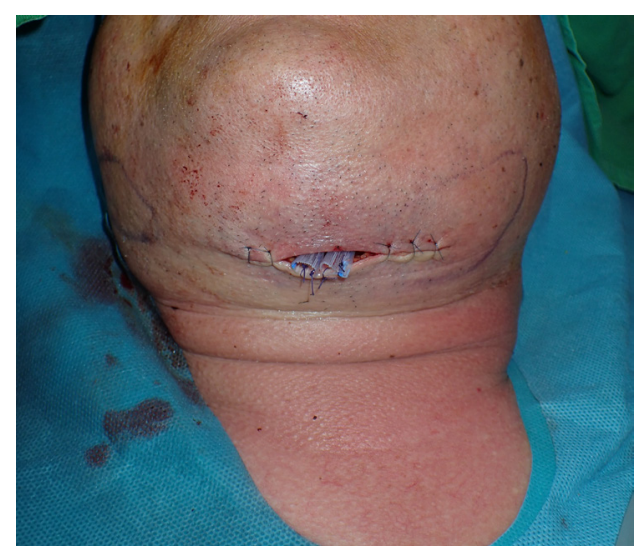

Fig. 5. Postoperative clinical photo: drain is seen on submental area.
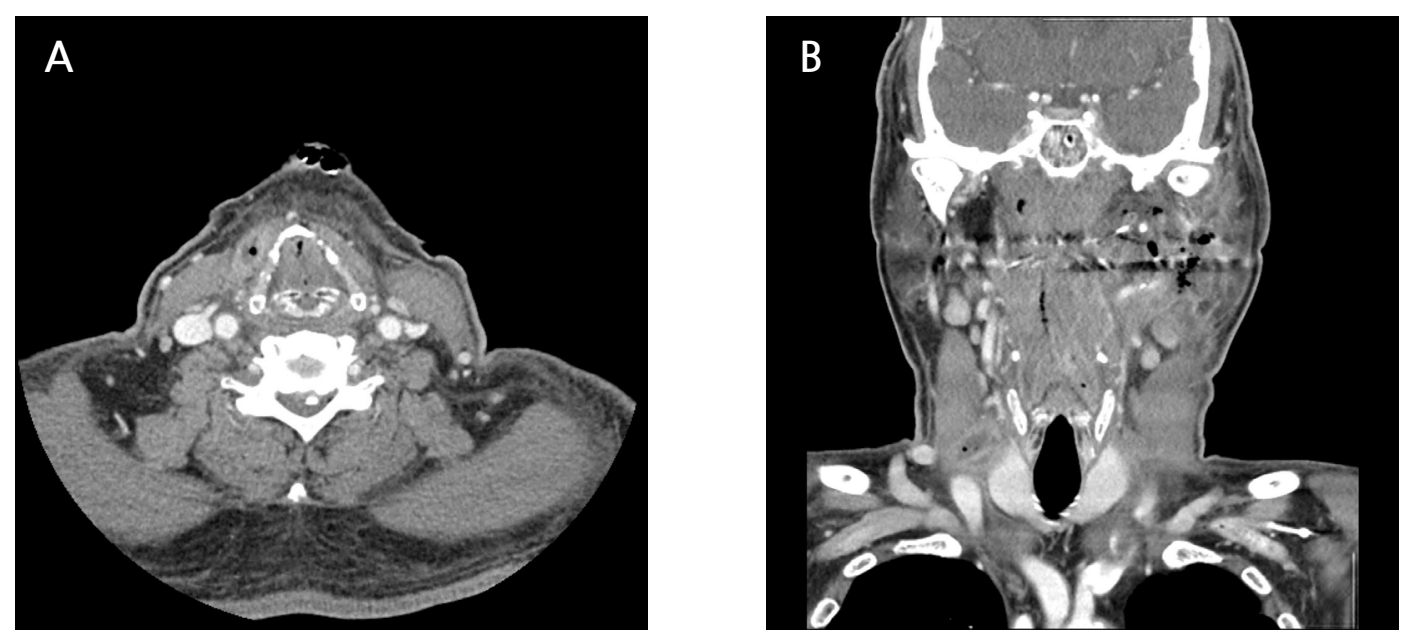

Fig. 6. Postoperative $C T$ view. Pus cavity in the right visceral space and left parapharyngeal space was seen from the neck CT. (A) axial view, (B) coronal view. 


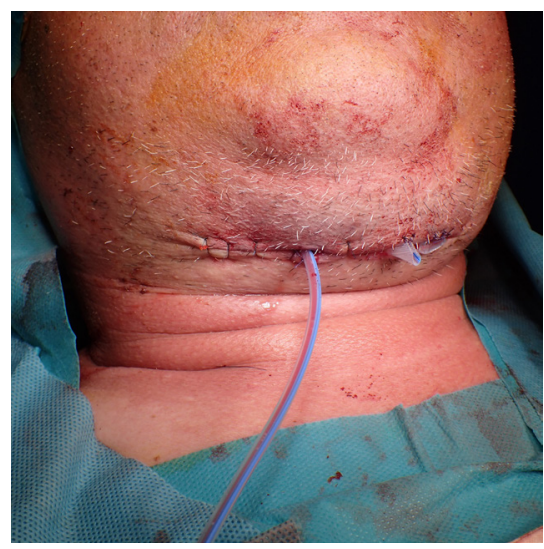

Fig. 7. Postoperative clinical photo: vacuum drain is seen on submental area.

Six days after the second surgery, most of the clinical symptoms resolved, and contrast-enhanced CT images confirmed that the pus cavity had disappeared. In addition, airway improvement was observed on postoperative CT. The laboratory test results showed a decrease in the CRP level to $0.9 \mathrm{mg} / \mathrm{dL}$, and treatment was completed.

During the initial hospitalization, ceftriaxone and metronidazole were administered, and the Streptococcus viridans group was detected on bacterial culture of pus collected during the primary surgery. Pseudomonas aeruginosa was detected in the pus cultured after the second surgery, and treatment was shifted to piperacillin/tazobactam.

\section{Discussion}

Most causes of fascial space infections are odontogenic, of which periodontal disease, apical lesions, and pericoronitis around the third molar are the most common. Although fascial space infection due to non-odontogenic causes is not common, it can be caused by infected needles, trauma, or salivary gland infection.

The space directly affected by the infection is called the primary fascial space, and the space affected by the spread of infection from the primary fascial space is called the secondary fascial space. The primary fascial spaces include the canine, buccal, and infratemporal spaces in the maxilla and submental space, buccal space, submandibular space, and sublingual space in the mandible. The secondary fascial spaces include the masseteric space, pterygomandibular space, and temporal space. If the infection spreads beyond the secondary fascial space, it also invades the deep cervical space, which can be lifethreatening. $^{2}$

Complications of odontogenic space infection include airway obstruction, sepsis, endocarditis, necrotizing fasciitis, spondylitis, brain abscess, thoracic emphysema, pleuropulmonary suppuration, 
aspiration pneumonia, pneumothorax, and mandibular or cervical osteomyelitis. ${ }^{3}$

Dental infection are caused by either apical lesions due to dental caries, fracture, etc., which result from bacterial invasion into the apical tissue following pulp necrosis, which is the most common route by which bacteria reach the bone directly; or periodontal causes, which occur because of bacterial invasion from the deep periodontal pocket into the underlying soft tissue. ${ }^{2}$ Infection occurs when the body's local defense mechanism is destroyed. The body's local defense mechanisms include the epidermis, mucous membrane, and normal resident bacteria. In the former, the anatomical barrier is destroyed through deep periodontal pockets and pulp necrosis, and bacteria can reach deeper. In the latter, the normal resident bacteria disappear or the composition ratio of resident bacteria changes because of the administration of antibiotics in patients with systemic diseases; hence, infections can occur easily.

The oral and maxillofacial infectious route largely depends on the location of the infection with respect to the fascia. The general rule is that infection spreads along the path of least resistance along the fascial plane, where the subcutaneous connective tissue and fascial layer can be separated. The purulent exudate collects at the location, forming the fascial space, and the fascial spaces communicate with each other, with infection spreading extensively and rapidly. ${ }^{4}$ Infectious spread is determined by the muscle attachment at the infection site, and most dental infections penetrate the bone through this route to form an abscess in the vestibule. If the infection spreads outside the muscle attachment, it affects the fascial space. ${ }^{5}$ Even if the infection starts locally, it spreads to the adjacent space via muscle movement, contraction, and relaxation, or to the paranasal sinuses, bloodstream, and lymphatic systems. ${ }^{6}$

Swelling caused by infection can narrow or deform airways. If there is a serious problem with the patient's breathing, preoperative endotracheal intubation is required. Even if preoperative intubation is not performed, intubation can be maintained after surgery, depending on the surgeon's predictions about infection control and airway changes during surgery. Compared to the airway deviation state on preoperative $\mathrm{CT}$, the patient clinically complained of respiratory discomfort before surgery. As a large amount of drainage occurred during the operation, it was possible to predict a significant improvement in the airway condition after the operation. Accordingly, postoperative extubation was immediately performed.

In this case, the common odontogenic causes mentioned above were unlikely to have led to fascial space infection, and other causes needed to be considered. First, the patient already had peri-implantitis, which refers to the destruction of hard and soft tissues surrounding the dental implant along with inflammation. When the infection is confined to the mucous membrane around the implant, it is called peri-implant mucositis. Since the attachment between the titanium surface of the implant and the connective tissue above the alveolar bone is weak, microbiological contamination that penetrates the 
bone easily occurs and destroys this attachment. ${ }^{7}$

Major risk factors for peri-implantitis include poor oral hygiene, dental cement residue, history of periodontitis, and smoking. Periodontal infection, overloading, and plaque deposition are the causes of delayed bone loss. According to Algraffee et al., ${ }^{8}$ cement residue should be considered a contributing factor for delayed peri-implant bone loss. Dental cement residues can cause mucositis and subsequently lead to bone loss as the inflammation spreads to the supporting bone, leading to chronic infection around the implant. In this case, the infection probably spread in a similar manner to that in cases in which fascial space infection occurs due to periodontal causes.

The second possible cause of the spread of infection is emphysema. Emphysema can occur in the presence of air pressure, which penetrates the fascial space of the tissue. ${ }^{9}$ In the absence of pressure, air cannot diffuse through the mucosal barrier. When air enters the tissue, the relatively soft connective tissue is separated from the adjacent muscle layer. Since the fascial spaces are interconnected, air from the mandible can diffuse to the retropharyngeal, mediastinal, and thoracic spaces. ${ }^{10}$ Microorganisms in the oral cavity can travel along the air inflow path, causing cellulitis or abscesses. Emphysema can also be induced by coughing, blowing strongly, smoking, or vomiting after dental treatment. Emphysema mainly results from the use of a high-speed air turbine drill during dental treatment, but can also result from instrument use, such as an air/water syringe. Due to the high pressure, air or debris can penetrate into the tissue, usually causing a local reaction and can reach the tissues deeper into the cervical fascial space. $^{11}$

In this case, both of the above causes seem to have worked together. During prosthesis recementation, an air/water syringe might be used repeatedly, and the microorganisms that existed in the vicinity of the implant owing to peri-implantitis were disseminated by air pressure, and possibly entered the fascial space, causing an infection. Additionally, the air bubble-filled cavity observed on the preoperative CT may occur because of the metabolism of anaerobes, or possibly, the emphysema. Because the patient's symptoms that appeared immediately after crown recementation treatment were not known, it was difficult to distinguish which one acted as the major contributing factor. Furthermore, systemic diseases such as diabetes might have contributed to delayed healing and more serious infections.

Treatment decisions are based on the cause and severity of the infection and the patient's current condition. The first line of treatment in cases of abscess is incision and drainage. In most cases, it can be performed under local anesthesia, and the most fluctuant site or the site where drainage can be performed smoothly owing to the effect of gravity is selected. An extraoral incision is required when the infection spreads rapidly through multiple fascial spaces or when it is difficult to perform smooth drainage through an intraoral incision. ${ }^{5}$

Antibiotic treatment is essential for controlling the causative strain of infection. Antibiotics were 
empirically selected at first; subsequently, the causative strain was cultured, and its susceptibility was tested, following which antibiotics were prescribed accordingly. ${ }^{12}$ The strains found in these infections have polymicrobial characteristics and are most susceptible to antibiotics such as amoxicillin/clavulanic acid, ceftriaxone, and levofloxacin, and metronidazole is known to have high sensitivity to mixed anaerobic strains commonly found in orofacial abscesses. ${ }^{13}$ In this case, $S$. viridans and $P$. aeruginosa were detected in the culture. $S$. viridans is a gram-positive and facultative anaerobic bacterium that is commonly found in odontogenic infections. Accordingly, ceftriaxone was selected as the first antibiotic, and metronidazole was added to counteract anaerobes, which are the common causative strains of advanced odontogenic infections. $P$. aeruginosa, which was detected in the culture after the second surgery, is a gram-negative bacterium resistant to third-generation cephalosporins. After consultation with an infectious disease specialist, the antibiotic was shifted to piperacillin/tazobactam.

When the acute phase of infection has subsided, it is necessary to eliminate the primary cause of infection to prevent recurrence. These include tooth extraction, root canal treatment, and the removal of pathological tissue. Although implant-related cases, such as the current case, have not been widely discussed, it is considered necessary to remove the implant suspected to be the cause or to observe the progress while treating peri-implantitis.

\section{IV . Conclusion}

Infections following non-invasive dental treatments have rarely been investigated. In this case, the abscess that occurred after recementation of the implant prosthesis had progressed to the right visceral space and left parapharyngeal space, extending to the superior area of the mandibular condyle. The cause of this infection seems to be the bacteria that existed around the implant owing to peri-implantitis and spread to the deep fascial space through a mechanism similar to the development of emphysema by air or water spray during treatment. In addition, due to a systemic disease such as diabetes, it might have progressed to a serious infection owing to delayed healing. Since serious fascial space infection is likely to occur even after non-invasive treatment related to dental implants, it is necessary to be aware of these infections.

\section{References}

1. Jung TY, Chae BM, Jeong YS, Park SJ. Retropharyngeal space abscess due to spread of odontogenic infection: two cases report. J Korean Assoc Oral Maxillofac Surg 2010;36:314-9.

2. Chang JS, Yoo KH, Yoon SH, Ha J, Jung S, Kook MS, et al. Odontogenic infection involving the 
secondary fascial space in diabetic and non-diabetic patients: a clinical comparative study. J Korean Assoc Oral Maxillofac Surg 2013;39:175-81.

3. Bali RK, Sharma P, Gaba S, Kaur A, Ghanghas P. A review of complications of odontogenic infections. Natl J Maxillofac Surg 2015;6:136-43.

4. Olsen I, van Winkelhoff AJ. Acute focal infections of dental origin. Periodontol 2000 2014;65:17889.

5. Ogle OE. Odontogenic Infections. Dent Clin North Am 2017;61:235-52.

6. Fehrenbach MJ, Herring SW. Spread of dental infection. Practical Hygiene 1997;6:13-9.

7. Bowen Antolin A, Pascua Garcia MT, Nasimi A. Infections in implantology: from prophylaxis to treatment. Med Oral Patol Oral Cir Bucal 2007;12:E323-30.

8. Algraffee H, Borumandi F, Cascarini L. Peri-implantitis. Br J Oral Maxillofac Surg 2012;50:689-94.

9. Jeong CH, Yoon S, Chung SW, Kim JY, Park KH, Huh JK. Subcutaneous emphysema related to dental procedures. J Korean Assoc Oral Maxillofac Surg 2018;44:212-9.

10. McKenzie WS, Rosenberg M. Iatrogenic subcutaneous emphysema of dental and surgical origin: a literature review. J Oral Maxillofac Surg 2009;67:1265-8.

11. Tan S, Nikolarakos D. Subcutaneous emphysema secondary to dental extraction: a case report. Aust Dent J 2017;62:95-7.

12. Dar-Odeh NS, Abu-Hammad OA, Al-Omiri MK, Khraisat AS, Shehabi AA. Antibiotic prescribing practices by dentists: a review. Ther Clin Risk Manag 2010;6:301-6.

13. Santosh AN, Viresh AN, Sharmada BK. Microbiology and antibiotic sensitivity of odontogenic space infection. Int J Med Dent Sci 2014;3:303-13. 